\title{
RESEARCH AND REPORTS
}

\section{Snapshot Prevalence and Characterization of Staphylococcus species, including MRSA, in a Student Athletic Facility: An Undergraduate Research Project}

\section{STEPHEN A. GARCIA, JACELLA F. MCKENZIE, THOMAS PATTERSON, RODNEY E. ROHDE}

OBJECTIVE: To evaluate and characterize the prevalence of MRSA, Staphylococcus aureus, and other Staphylococcus species found on exercise equipment on one day point of collection.

DESIGN: A cross sectional, point prevalence design (pilot study) with a single time of measurement. Data collected in summer of 2011 (Undergraduate Research Project for CLS Program, CLS 4361 Clinical Research). Project received Institutional Review Board exemption.

SETTING: In a higher education athletic facility in Texas.

SAMPLES: One hundred twenty-five environmental samples were collected from various exercise equipment and areas within the facility.

MAIN OUTCOME MEASURES: Samples were screened for Staphylococcus species using standard microbiological techniques. Confirmation of $S$. aureus was conducted by DRYSPOT Staphytect Plus ${ }^{\mathrm{TM}}$. MRSA isolates were confirmed with CHROMagar ${ }^{\mathrm{TM}}$; VITEK $^{\circ}$ 2 antibiotic susceptibility testing and PFGE characterized all MRSA isolates.

RESULTS: Prevalence of MRSA was 6\%, S. aureus $38 \%$, other Staphylococcus species 52\% and no growth $4 \%$. Prevalence of $S$. aureus and MRSA was highest on free weights and mats, respectively. PFGE characterized all MRSA isolates as HA-MRSA (USA100 strain).

CONCLUSIONS: Although limitations exist for this study with a single time of measurement for data, the findings indicate potential exposure risks from Staphylococcus species in college athletic facilities. Compliance (disinfection) and creative health education may reduce transmission of pathogens, environmental load, and incidence of colonization or infection in students.

ABBREVIATIONS: CA-MRSA - Community-associated methicillin resistant Staphylococcus aureus; CLS Clinical Laboratory Science; HAIs - Healthcare associated infections; HA-MRSA - Healthcare-associated methicillin resistant Staphylococcus aureus; MDRO Multiple Drug Resistant Organisms; MRSA Methicillin resistant Staphylococcus aureus.

INDEX TERMS: antibiotic resistance, CA-MRSA, college health, environmental contamination, MRSA, Multiple Drug Resistant Organisms, skin infections, Staphylococcus aureus

Clin Lab Sci 2012;25(3):156

Stephen A. Garcia, BSCLS, MLS (ASCP) ${ }^{C M}$, College of Health Professions, Clinical Laboratory Science Program Texas State University, San Marcos, TX

Jacella F. McKenzie, BSCLS, MLS (ASCP) ${ }^{C M}$, College of Health Professions, Clinical Laboratory Science Program Texas State University, San Marcos, TX

Thomas Patterson, MS, MT(ASCP), College of Health Professions, Clinical Laboratory Science Program Texas State University, San Marcos, TX

Rodney E. Rohde, PhD, MS, SV, SM(ASCP) ${ }^{C M} M B^{C M}$, College of Health Professions, Clinical Laboratory Science Program, Texas State University, San Marcos, TX

Address for Correspondence: Rodney E. Rohde, PhD, $M S, S V, S M(A S C P)^{C M} M B^{C M}$, Associate Dean of Research 
\& Associate Professor, College of Health Professions, Texas State University, Clinical Laboratory Science, HPB 361, 601 University Drive, San Marcos, TX 78666-4616, 512-245-2562, rrohde@txstate.edu

\section{INTRODUCTION}

Staphylococcus aureus is a gram positive bacterium commonly found on the skin and in the nose of 25$30 \%$ of healthy individuals. The bacterium can cause infection by entering the body through a cut, sore, breathing tube, or catheter. It often causes skin infections however it can also cause more serious infections such as sepsis, pneumonia, endocarditis, or osteomyelitis in immunocompromised individuals. Methicillin resistant Staphylococcus aureus (MRSA) is a strain of $S$. aureus that is currently resistant to all available $\beta$-lactam antibiotics such as oxacillin, a derivative of penicillin, and cephalosporins. ${ }^{1}$ Almost two million people a year in the United States acquire a MRSA infection with approximately 99,000 resulting in death. ${ }^{2}$ The annual health care costs for MRSA infections total nearly $\$ 4.5$ billion a year. ${ }^{3}$ According to the Centers for Disease Control (CDC), MRSA infections are grouped into two types: Healthcare Associated MRSA (HA-MRSA) and Community Associated MRSA (CA-MRSA). HA-MRSA infections generally occur in people who are or have recently been in a hospital or other health-care facility. Americans visit the doctor approximately 12 million times each year to get checked for suspected Staphylococcus species or MRSA skin infections. CA-MRSA infections generally occur in otherwise healthy people who have not been hospitalized or undergone a medical procedure within the past year. ${ }^{4}$ Studies over the past decade have determined that isolates causing HA-MRSA and CAMRSA infections have distinct strains. The two types of MRSA should be properly identified through laboratory typing tools such as pulse field gel electrophoresis (PFGE) or sequence typing. Although HA-MRSA infections are much more predominant than CAMRSA, the community associated strain has become the most frequent cause of skin and soft tissue infections presenting to emergency rooms in the United States; it appears that more people in the United States now die from MRSA, especially HA-MRSA, than from HIV/AIDS. ${ }^{4-5}$

Athletes, who participate in organized and recreational sports, and individuals who work out solely in athletic facilities, are uniquely susceptible to skin infections. The historical conditions "jock itch" and "athlete's foot" have long lent themselves to this understanding. Staphylococcal skin infections, such as impetigo and furuncles, are not new to sports teams $s^{6-7}$ but were rarely linked to major headlines since they could easily be treated with common beta lactam antibiotics such as penicillin. More recently however, a public health storm has been on a collision course with contact sports and athletes because of the emergence of a very real threat MRSA. In the past 15 years, MRSA debuted in contact team sports ${ }^{8-9}$ and many other sectors of society (e.g. jails, universities, IV drug users). ${ }^{10-14}$ Universities and their natural link to a variety of organized and intramural sports, as well as recreational athletic facilities, make them a worrisome environment of these types of highly transmissible pathogens. MRSA has distinguished itself as a highly virulent agent in previously healthy young people and therefore has become a worthy and fearful adversary in the world of academic settings, as well as the general community.

MRSA infections have been linked to athletic facilities due to the sharing of contaminated exercise equipment. Previous studies have revealed that MRSA is able to survive on a wide range of surfaces in a high school wrestling environment for extended periods of time and can infect hosts even with limited exposure ${ }^{3}$ as well as among a college football team with outbreak history. ${ }^{15}$ In a study of Texas $4 \mathrm{~A}$ and $5 \mathrm{~A}$ high school athletic departments, licensed athletic trainers reported MRSA in their athletic departments. The study reported the largest outbreak occurred in football players (23 infected) and the trainers also indicated that MRSA was found in wrestlers, volleyball players, cross-country runners, non-athlete students, and adults. ${ }^{16}$ Interestingly, MRSA seems to have no limit in its reach regarding athletic venues (e.g. fencing, all levels of high school, college, and professional arenas). ${ }^{15,17-20}$

Pathogens such as MRSA, Clostridium difficile, and vancomycin resistant enterococci (VRE) have been shown to remain viable for extended periods of time on surfaces. For instance, MRSA strains can remain viable for up to two weeks on Formica surfaces, and for up to six to nine weeks on cotton-fabrics. ${ }^{21-23}$ Some outbreak strains of MRSA have been shown to have longer survival rates with higher concentrations than nonoutbreak strains. ${ }^{21,24}$ In an interesting study conducted 
by Colbeck, ${ }^{25}$ it was shown that $S$. aureus can remain virulent and capable of causing infection for at least 10 days after exposure to dry surfaces. The transmission of bacteria is known to occur by hand contact with fomites, therefore exercise equipment and other high contact areas may serve as "hot spot" reservoirs in athletic facilities.

The overall aim in this research project had three objectives: first, to assess the prevalence of $S$. aureus and MRSA in an athletic facility within an academic setting to better characterize the microbial environment and the potential for transmission; second, to identify potential risks associated with certain areas, environments or types of equipment found in an athletic facility; and third, to provide an opportunity for CLS students to participate in an educational undergraduate research project. The information provided in this study should allow for a better understanding among various health professionals that work with college-aged students (eg, physicians, nurses, medical laboratory scientists) as well as university and public health officials who plan and implement campus health policies, especially regarding MDROs such as MRSA.

\section{MATERIALS AND METHODS}

\section{Study Design}

In this cross-sectional pilot study of a university athletic facility's microbial environment for Staphylococcus species, a standardized $(4 \mathrm{~cm} \times 4 \mathrm{~cm})$ hand contact area was sampled using a culturette swab containing Amies Liquid medium (BBL Microbiology Systems; Becton Dickinson) from a variety of recreational equipment (eg, treadmills, hand weights, bikes, etc.) and areas (eg, mats, hand scanners, etc). Consultation and permission was obtained with the athletic facility director for the study. Additionally, the research project was granted exempt status from the Institutional Review Board because the study did not involve human subjects and was totally anonymous. Finally, this study was conducted as a component of a clinical laboratory science (CLS) course project (CLS 4361: Clinical Research) for part of the requirements towards a BSCLS degree.

The athletic facility is a typical recreational environment that one might find in any academic institution. This particular facility has the following amenities: aquatics, weight room, indoor track, racquetball, gyms, specialized exercise rooms (eg, yoga, aerobics, etc.) and a variety of other features (eg, showers, changing areas, classrooms, etc.). Due to the research project being conducted as part of an undergraduate course, the investigators chose to utilize a single day of sampling (point prevalence) with a random sampling of equipment and areas in the high-throughput region of the weight room. Annual entrances into the facility (April 12, 2010 - April 11, 2011) were 582,562. The monthly total for entrances (March 12, 2011 - April 11, 2011, includes March 12 and April 11) was 54,701. The sampling day total for this research project (April 11,2011 ) was 2,008 individuals.

\section{Laboratory Analysis}

A total of 125 samples were collected from the following exercise equipment as well as other areas of interest in the recreational facility: Ellipticals $(\mathrm{N}=25)$, bikes $(\mathrm{N}=25)$, machine weights $(\mathrm{N}=25)$, free weights $(\mathrm{N}=25)$, treadmills $(\mathrm{N}=5)$, mats $(\mathrm{N}=5)$, medicine balls $(\mathrm{N}=5)$, basketballs $(\mathrm{N}=5)$, hand scanners $(\mathrm{N}=3)$, and rowing machines $(\mathrm{N}=2)$. A culturette swab (BBL Microbiology Systems; Becton Dickinson) containing Amies Liquid Medium was used to swab the handcontact surface area of the exercise equipment and other areas of interest. Samples were screened for Staphylococcus species using the standard screening media, mannitol salt agar (MSA), Dry Spot Staphytect Plus test kits (Oxoid Limited, Lenexa, KS), and Dropit catalase reagent (Key Scientific Products, Round Rock, TX). The $7.5 \%$ concentration of sodium chloride in MSA (Becton Dickinson BBL, Franklin Lakes, NJ) results in the partial or complete inhibition of bacterial organisms other than Staphylococcus species. Mannitol fermentation, as indicated by a color change of the phenol red indicator to yellow, aids in the differentiation of Staphylococcus species. Typical colony morphology of $S$. aureus on MSA is small to large colonies with yellow zones.

After incubation at $35 \pm 2^{\circ} \mathrm{C}$ for 48 hours, suspected $S$. aureus colonies on MSA were tested with the DRYSPOT Staphytect Plus ${ }^{\mathrm{TM}}$ (Oxoid) which is a latex slide agglutination test for the differentiation of $S$. aureus by detection of clumping factor, Protein A and certain polysaccharides found in MRSA from other Staphylococcus species that do not possess these properties. Suspected $S$. aureus isolates identified using 
the DRYSPOT Staphytect Plus ${ }^{\mathrm{TM}}$ were confirmed by CHROMagar $^{\mathrm{TM}}$ MRSA Select (BBL Microbiology Systems; Becton Dickinson). CHROMagar ${ }^{\mathrm{TM}}$ is a chromogenic medium used for the isolation and differentiation of MRSA. MRSA strains will grow in the presence of cefoxitin and hydrolyze the chromogenic substrate to produce rose to mauve-colored colonies. Additionally, positive colony growth on CHROMagar $^{\mathrm{TM}}$ was confirmed as MRSA by Vitek 2 (bioMérieux, Hazelwood, MO) susceptibility testing using Vitek GN19 susceptibility cards. Cards were inoculated and incubated in the Vitek 2 per manufacturer recommendations and results were analyzed by the advanced expert system, software version R04.03. All tests were performed according to the manufacturer's instructions. All growth on MSA or CHROMagar $^{\mathrm{TM}}$ not consistent with $S$. aureus or MRSA was resulted as "other" Staphylococcus species. S. aureus, MRSA, and $S$. epidermidis specimens were provided by an affiliated clinical laboratory, as confirmed by Vitek 2 analysis, and were used as positive and negative controls during inoculation of all microbiological testing

\section{Antibiotic Susceptibility Testing}

Each MRSA isolate was tested for susceptibility, intermediate resistance, or resistance to a panel of antibiotics. Susceptibility testing was conducted by the Vitek 2 automated microbiology system (per standard manufacturer protocol) and data was examined for any unique antibiotic resistance trends or outliers as identified by standardized results from the manufacturer.

\section{Molecular Typing}

Any confirmed MRSA isolates were sent to the Texas Department of State Health Services (DSHS) to perform pulsed-field gel electrophoresis (PFGE) to determine the strain type as recommended by DSHS standard methodology. The standard control for PFGE on MRSA isolates was NCTC8325. The dendogram of the PFGE pattern was sent to the Centers for Disease Control and Prevention (CDC) to confirm the MRSA strain as compared to the national MRSA PFGE database.

\section{RESULTS}

Descriptive Analyses

A multitude of Staphylococcus species were detected on the hand contact surfaces of the exercise equipment swabbed. This study focused on samples positive for $S$. aureus and further testing was performed within those samples for detection of MRSA. Out of the 125 samples collected, a total of 55 colonies were identified as $S$. aureus with the DRYSPOT Staphytect Plus ${ }^{\mathrm{TM}}$. Those colonies were subsequently subcultured onto CHROMagar $^{\mathrm{TM}}$ for identification of MRSA strains. Out

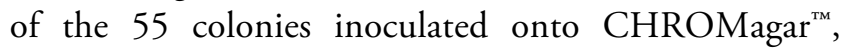
seven colonies were identified as MRSA ( $S$. aureus $\mathrm{N}=48$, MRSA $\mathrm{N}=7$ ). The overall prevalence for the 125 samples that were collected is as follows: no growth 5 (4\%), MRSA 7 (6\%), S. aureus $48(38 \%)$, and other Staphylococcus species 65 (52\%). Table 1 shows the complete results for each set of exercise equipment and area swabbed and its corresponding Staphylococcus species prevalence. $S$. aureus prevalence was highest on free weights and MRSA prevalence was highest on mats.

Table 1. Staphylococcus species prevalence on equipment and areas.

\begin{tabular}{lccccc}
\hline Item & $\begin{array}{c}\text { Total } \\
\text { Swabbed }\end{array}$ & (NG/-) & MRSA (+) & S. aureus (+) & $\begin{array}{c}\text { Other } \\
\text { staph (+) }\end{array}$ \\
Bikes & 25 & $1(4 \%)$ & $1(4 \%)$ & $6(24 \%)$ & $17(68 \%)$ \\
Ellipticals & 25 & $3(12 \%)$ & $2(8 \%)$ & $4(16 \%)$ & $16(64 \%)$ \\
Machine wts. & 25 & 0 & $1(4 \%)$ & $9(36 \%)$ & $15(60 \%)$ \\
Free wts. & 25 & 0 & $1(4 \%)$ & $20(80 \%)$ & $4(16 \%)$ \\
Mats & 5 & 0 & $2(40 \%)$ & $3(60 \%)$ & 0 \\
Medicine balls & 5 & 0 & 0 & $2(40 \%)$ & $3(60 \%)$ \\
Basketballs & 5 & 0 & 0 & $3(60 \%)$ & $2(40 \%)$ \\
Treadmills & 5 & 0 & 0 & $1(20 \%)$ & $4(80 \%)$ \\
Hand scanners & 3 & 0 & 0 & 0 & $3(100 \%)$ \\
Row machines & 2 & $1(50 \%)$ & 0 & 0 & $1(50 \%)$ \\
Total & 125 & $5(4 \%)$ & $7(6 \%)$ & $48(38 \%)$ & $65(52 \%)$
\end{tabular}

NG/- is no growth or negative; MRSA is methicillin-resistant Staphylococcus aureus; + is positive growth; Other staph is species other than $S$. aureus

\section{Antibiotic Susceptibility and Molecular Characterization}

Antibiotic susceptibility characteristics of MRSA isolates are shown in Table 2. Each MRSA isolate (seven) was analyzed for antibiotic susceptibility. Virtually identical patterns were observed for each isolate with a few exceptions (ciprofloxacin, gentamicin, and levofloxacin). Beta-lactamase and cefoxitin screens were positive for each MRSA isolate; all isolates were 100\% resistant to beta lactam antibiotics. Vancomycin, linezolid, moxifloxacin, quinupristin/dalfopristin, tetracycline, tigecycline, and rifampicin were $100 \%$ effective (susceptible). Interestingly, erythromycin and imipenem was $100 \%$ resistant to all isolates.

The seven isolates identified as MRSA were sent to the DSHS for analysis of clonal similarities using PFGE. 
After analysis, the seven isolates were determined to have an identical PFGE profile. An image (dendogram)

Table 2. Antibiotic susceptibility of methicillin-resistant Staphylococcus aureus (MRSA) isolates.

\begin{tabular}{|c|c|c|c|c|c|c|c|c|c|c|c|c|c|}
\hline \multirow[b]{2}{*}{$\mathrm{Abx}^{\mathrm{A}}$} & \multicolumn{8}{|c|}{ Isolates $^{\mathrm{B}}$} & \multicolumn{5}{|c|}{$\mathrm{RXN}^{\mathrm{C}}$} \\
\hline & 15 & 31 & 47 & 72 & 95 & 103 & 105 & & $\% \mathrm{~S}$ & $\% \mathrm{R}$ & $\% \mathrm{I}$ & $\% \mathrm{P}$ & $\% \mathrm{~N}$ \\
\hline $\mathrm{BL}$ & $\mathrm{P}$ & $\mathrm{P}$ & $\mathrm{P}$ & $\mathrm{P}$ & $\mathrm{P}$ & $\mathrm{P}$ & $\mathrm{P}$ & & & & 100 & 0 & \\
\hline CFS & $\mathrm{P}$ & $\mathrm{P}$ & $\mathrm{P}$ & $\mathrm{P}$ & $\mathrm{P}$ & $\mathrm{P}$ & $\mathrm{P}$ & & & & 100 & 0 & \\
\hline ICR & $\mathrm{N}$ & $\mathrm{N}$ & $\mathrm{N}$ & $\mathrm{N}$ & $\mathrm{N}$ & $\mathrm{N}$ & $\mathrm{N}$ & & & & 0 & 100 & \\
\hline $\mathrm{AZ}$ & $\mathrm{R}$ & $\mathrm{R}$ & $\mathrm{R}$ & $\mathrm{R}$ & $\mathrm{R}$ & $\mathrm{R}$ & $\mathrm{R}$ & 0 & 100 & 0 & & & \\
\hline BP & $\mathrm{R}$ & $\mathrm{R}$ & $\mathrm{R}$ & $\mathrm{R}$ & $\mathrm{R}$ & $\mathrm{R}$ & $\mathrm{R}$ & 0 & 100 & 0 & & & \\
\hline CFA & $\mathrm{R}$ & $\mathrm{R}$ & $\mathrm{R}$ & $\mathrm{R}$ & $\mathrm{R}$ & $\mathrm{R}$ & $\mathrm{R}$ & 0 & 100 & 0 & & & \\
\hline CFO & $\mathrm{R}$ & $\mathrm{R}$ & $\mathrm{R}$ & $\mathrm{R}$ & $\mathrm{R}$ & $\mathrm{R}$ & $\mathrm{R}$ & 0 & 100 & 0 & & & \\
\hline CFT & $\mathrm{R}$ & $\mathrm{R}$ & $\mathrm{R}$ & $\mathrm{R}$ & $\mathrm{R}$ & $\mathrm{R}$ & $\mathrm{R}$ & 0 & 100 & 0 & & & \\
\hline CFU & $\mathrm{R}$ & $\mathrm{R}$ & $\mathrm{R}$ & $\mathrm{R}$ & $\mathrm{R}$ & $\mathrm{R}$ & $\mathrm{R}$ & 0 & 100 & 0 & & & \\
\hline CIP & $\mathrm{R}$ & $\mathrm{R}$ & $\mathrm{R}$ & $\mathrm{R}$ & $S$ & I & $S$ & 29 & 57 & 14 & & & \\
\hline CLA & $\mathrm{R}$ & $\mathrm{R}$ & $\mathrm{R}$ & $\mathrm{R}$ & $\mathrm{R}$ & $\mathrm{R}$ & $\mathrm{R}$ & 0 & 100 & 0 & & & \\
\hline CLIN & $\mathrm{R}$ & $\mathrm{R}$ & $\mathrm{R}$ & $\mathrm{R}$ & $\mathrm{R}$ & I & $\mathrm{R}$ & 0 & 86 & 14 & & & \\
\hline ERY & $\mathrm{R}$ & $\mathrm{R}$ & $\mathrm{R}$ & $\mathrm{R}$ & $\mathrm{R}$ & $\mathrm{R}$ & $\mathrm{R}$ & 0 & 100 & 0 & & & \\
\hline GEN & $\mathrm{R}$ & $S$ & $\mathrm{R}$ & $\mathrm{R}$ & $S$ & I & $S$ & 43 & 43 & 14 & & & \\
\hline IMP & $\mathrm{R}$ & $\mathrm{R}$ & $\mathrm{R}$ & $\mathrm{R}$ & $\mathrm{R}$ & $\mathrm{R}$ & $\mathrm{R}$ & 0 & 100 & 0 & & & \\
\hline $\mathrm{LF}$ & I & $S$ & I & I & $S$ & $S$ & $S$ & 57 & 0 & 43 & & & \\
\hline $\mathrm{LZ}$ & $S$ & $S$ & $S$ & S & $S$ & $S$ & $S$ & 100 & 0 & 0 & & & \\
\hline MXF & $S$ & $S$ & $S$ & $S$ & $S$ & $S$ & $S$ & 100 & 0 & 0 & & & \\
\hline NF & $S$ & $S$ & $S$ & $S$ & $S$ & I & $S$ & 86 & 0 & 14 & & & \\
\hline OX & $\mathrm{R}$ & $\mathrm{R}$ & $\mathrm{R}$ & $\mathrm{R}$ & $\mathrm{R}$ & $\mathrm{R}$ & $\mathrm{R}$ & 0 & 100 & 0 & & & \\
\hline $\mathrm{Q} / \mathrm{DF}$ & $S$ & $S$ & $S$ & $S$ & $S$ & $S$ & $S$ & 100 & 0 & 0 & & & \\
\hline $\mathrm{R}$ & $S$ & $S$ & $S$ & $S$ & $S$ & $S$ & $S$ & 100 & 0 & 0 & & & \\
\hline TET & $S$ & $S$ & $S$ & $S$ & $S$ & $S$ & $S$ & 100 & 0 & 0 & & & \\
\hline TIG & $S$ & $S$ & $S$ & $S$ & $S$ & $S$ & $S$ & 100 & 0 & 0 & & & \\
\hline TMP & $\mathrm{R}$ & $S$ & $\mathrm{R}$ & $\mathrm{R}$ & $\mathrm{R}$ & $S$ & $\mathrm{R}$ & 29 & 71 & 0 & & & \\
\hline VAN & $S$ & $S$ & $S$ & $S$ & $S$ & $S$ & $S$ & 100 & 0 & 0 & & & \\
\hline
\end{tabular}

A. Abbreviated antibiotics: $\mathrm{BL}=$ beta-lactamase screen; $\mathrm{CFS}=$ cefoxitin screen; $\mathrm{ICR}=$ inducible clindamycin screen; $\mathrm{AZ}=$ azithromycin; $\mathrm{BP}=$ benzylpenicillin; CFA = cefaclor; $\mathrm{CFO}=$ cefotaxime; $\mathrm{CFT}=$ ceftriaxone; $\mathrm{CFU}=$ cefuroxime; $\mathrm{CIP}=$ ciprofloxacin; CLA = clarithromycin; CLIN = clindamycin; ERY = erythromycin; GEN = gentamicin; IMP = imipenem; LF = levofloxacin; $\mathrm{LZ} \mathrm{=} \mathrm{linezolid;} \mathrm{MXF}=$ moxifloxacin; $\mathrm{NF}=$ nitrofurantoin; $\mathrm{OX}=$ oxacillin; $\mathrm{Q} / \mathrm{DF}=$ quinupristin/dalfopristin; $\mathrm{R}$ = rifampicin; TET = tetracycline; TIG tigecycline; $\mathrm{TMP}=$ trimethoprim; $\mathrm{V}=$ vancomycin

B. Isolate numbers (Seven total MRSA isolates; $\mathrm{P}=$ positive, $\mathrm{N}=$ negative; $\mathrm{S}=$ susceptible, $\mathrm{R}=$ resistant, $\mathrm{I}=$ intermediate

C. $\mathrm{RXN}=$ Total percentage of MRSA isolates identified as $\mathrm{S}, \mathrm{R}$ or I

of the PFGE profile was sent to the CDC for comparison with the CDC MRSA Isolate Pattern Database. Similarity was determined by cluster analysis using BioNumerics software. According to the CDC, the pattern of all seven MRSA isolates identified in this study matched most frequently with strain USA100 pattern in their database. The USA100 strain is one of the more common HA-MRSA strains. ${ }^{5}$ Figure 1 illustrates the PFGE patterns that were identified at DSHS and confirmed with the CDC.

\section{DISCUSSION}

This cross sectional study of Staphylococcus species microbial environment in an athletic facility of a university revealed the following overall point prevalence of $S$. aureus (48/125, 38\%), MRSA (7/125, 6\%), and other Staphylococcus species (65/125, 52\%). These findings support the existence of a potential risk of coming into contact with $S$. aureus, MRSA, or other Staphylococcus species in this athletic facility based on 2,008 individuals that entered the facility on the sampling day for this research project (April 11, 2011).

Several studies have examined the community prevalence of MRSA found on equipment used in competitive contact sports such as football, wrestling, and rugby (refs) but few studies have examined the overall prevalence of MRSA found on recreational exercise equipment in an athletic facility. ${ }^{26} \mathrm{With}$ respect to equipment, $S$. aureus prevalence ranged from $0-80 \%$ 
in this study. Interestingly, free weights had the highest $S$. aureus prevalence $(20 / 25,80 \%)$; mats were highest for MRSA prevalence $(2 / 5,40 \%)$. This finding may be attributed to the nature of free weights having a greater likelihood of creating abrasions and lacerations or, in the case of non-equipment such as mats, that there is a high contact area with skin. Our data supports intuition, anecdotal observations, and investigational findings that physical contact sports represent the greatest risk because of the opportunity for pathogens, especially MRSA, to pass directly from person to person via abrasions and lacerations which create a portal of entry. ${ }^{16}$ Here, we show that using exercise equipment and environmental areas with high skin contact as solitary events (non-team sports) within a recreational athletic center also may indicate a risk for Staphylococcus species exposure. This also supports the findings of outbreak investigations that MRSA (and potentially $S$. aureus and other "staph infections") exposures are occurring in minimal-contact situations. ${ }^{17,19}$

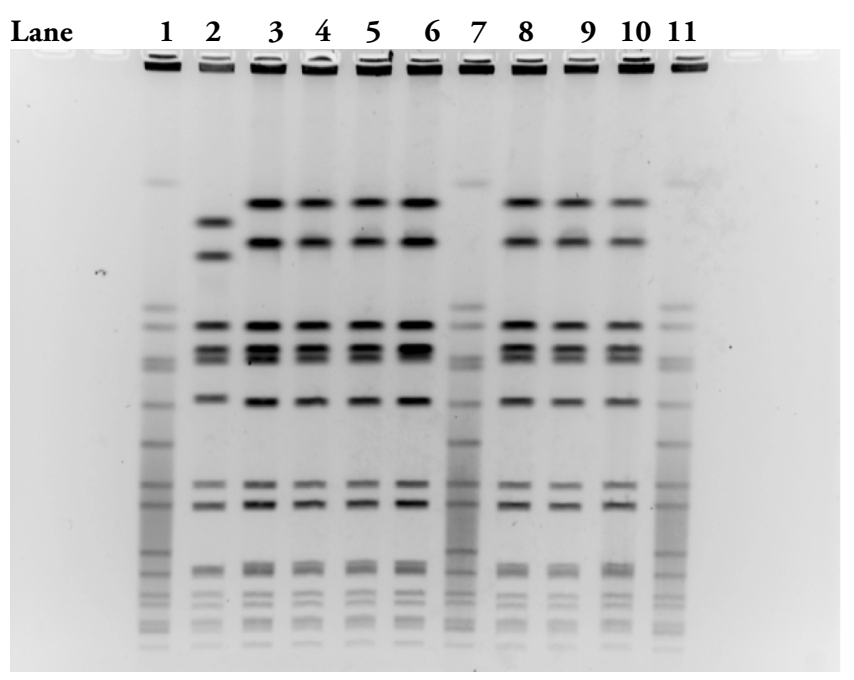

Figure 1. Pulse Field Gel Electrophoresis (PFGE) profiles of MRSA Isolates. Lane 1, 7 and 11 are controls and lane 2 is a specimen not submitted from this study. Standard control = NCTC8325. Lanes 3-6 and 8-10 illustrate identical patterns to USA100 strain (HA-MRSA) as compared to CDC MRSA Isolate Pattern Database.

Further studies (longitudinal/cohort and/or case control) of other recreational exercise and athletic facilities should be conducted to more accurately assess the colonization and infection risk involved with users of exercise equipment and high skin contact areas within these types of facilities. Typically, most nationally representative surveys of nasal colonization with $S$. aureus find the prevalence of nasal colonization with $S$. aureus to be $28.6 \%$ and MRSA $1.5 \% .{ }^{1}$ Recently, nasal carriage rates of $S$. aureus and MRSA were reported in a university student population to be $29.6 \%$ and $7.4 \%$ respectively. It was also found that hospitalization within the past 12 months and recent skin infection was associated with being a MRSA carrier in the student population. ${ }^{11}$ Interestingly, in this study of an athletic facility, PFGE analysis showed all seven MRSA isolates to be of the same strain, the HA-MRSA USA100 strain which is typically a healthcare associated strain of MRSA. This could be an indication that a user(s) of the athletic equipment may have been in a hospital or other health-care facility. However, this may also be an indication that the lines between HA-MRSA and CA-MRSA are beginning to overlap within the community including academic environments. Perhaps, HA-MRSA is being transmitted serially within the general community, such as a university setting, instead of solely in the silo environment of a particular healthcare facility. As mentioned previously, it has been documented that MRSA, as well as other MDROs, can remain virulent and capable of causing infection for at least 10 days on dry surfaces, 14 days on formica surfaces, and up to six to nine weeks on cotton-blanket material. ${ }^{21}$ These findings, together, bring into sharp focus that while shared athletic facility items (exercise equipment, mats, etc.) potentially serve as fomites in Staphylococcus species transmission (including MRSA), classroom and social contact (dormitories, intramural events, etc.) among students may also play a significant role.

\section{Limitations}

The greatest limitation of this study is the nature of determining transmission to an individual. Due to the nature of the study being incorporated into an undergraduate research experience per a course requirement, the research design chosen was a single day for point prevalence of Staphylococcus species. This limitation does not allow for a longitudinal approach with respect to environmental cleaning effect over time, nor does it allow tracking actual colonization or infection among single users of equipment or areas within the facility. That approach would be important to build data supporting risk factors associated with $S$. aureus and MRSA, as well as transient flora issues (environment microbial load) on equipment or areas within an athletic facility. We are only able to report 
prevalence on particular equipment and areas at a particular point in time. Additionally, due to limited resources per an undergraduate project, the number of sampled items (N) was quite low for several items. Future work in this type of environment would necessitate a greater $\mathrm{N}$ for statistical power and broad generalization to similar environments.

\section{CONCLUSIONS AND RECOMMENDATIONS}

Today's college and/or university settings have been impacted by many changes with respect to athletic and recreational facilities over the past 20-30 years. The days of mortar and brick buildings, hidden in some corner of the campus, housing only a few basketball courts or weight rooms are long gone. Trends have shifted dramatically on campus with respect to the intersection of demographics, integration of curriculum and health performance, and diversity of culture around campus recreation facilities. ${ }^{27}$ Females, for instance, now make up around $75 \%$ of the university population ${ }^{28}$ which has influenced the explosion towards "fitness" areas versus only the traditionally male-dominated gym and weight rooms. Likewise, the modern college student utilizes the campus athletic/recreational facility as a social networking environment. Students expect the environment to be social friendly towards electronicsharing, dining, and being "green" (rock walls, environmentally sound, etc.) as they move easily in and out of these open-platform styles of facility construction/renovation. ${ }^{27}$

With these new trends in mind, the modern college student demands our attention with respect to their general health and how the new social environment plays out in the world of newly emerging and reemerging antibiotic resistant organisms, as well as other resistant microbes (e.g. influenza, meningitis, foodborne illness such as listeriosis). Casual recreation and exercise by college students is not confined to the athletic facility. Contrary to popular belief that microbes remain in facilities (healthcare, gyms, restaurants, etc.), individuals and their personal belongings are essentially vectors of transmission as they interact with their classmates outside the recreational/athletic facility, family and friends within the general community, and other individuals in their daily lives. All of which may be widely separated over time and geographic locales. Thus, these individuals must be targeted for intervention because of the potential for dissemination of MRSA and other like microbes is strong.

This study was simply a point prevalence of standard exercise equipment and areas of a typical athletic facility found at a typical university. However, we have shown that in a typical day of this type of facility one might find the prevalence of Staphylococcus species to be significant (Table 1). This study is not an indictment or a "finger pointing" study towards university athletic facilities or similar environments, private or public. Rather, it's an effort to heighten awareness on college campuses with respect to SOPs for environmental disinfection and health policy for students, faculty, and staff. We recommend that shared equipment that comes into direct skin contact should be cleaned before and after each use with attention given to enforced contact time of agent and other requirements for effectiveness. The CDC recommends cleaning surfaces with detergent-based cleaners or disinfectants registered by the Environmental Protection Agency to effectively remove MRSA from the environment. Therefore, routine cleaning and disinfection of contaminated environmental surfaces can reduce the transmission of pathogens and the incidence of colonization or infection. ${ }^{4}$ Importantly, new methods and applications are currently being evaluated to reduce environmental microbial loads (e.g. $\mathrm{H}_{2} \mathrm{O}_{2}$, fluorescent gel, ATP system, etc.). ${ }^{29}$

Likewise, an effort to educate all those involved with the health and well-being of university populations as it relates to Staphylococcus species, including MRSA, should be enacted nationwide. Indeed, a recent study about the knowledge, learning, and adaptation of the general public towards MRSA has shown severe gaps in critical understanding. Individuals need guidance with respect to obtaining proper MRSA prevention, diagnosis, and adaption to stigmatization around this infection. Of most importance is the need for the general population, including college students, to be taught the importance of a MRSA diagnosis from a culture and antibiotic susceptibility test. ${ }^{30}$

In light of our study (and similar studies mentioned previously) and a recent study showing a 7.4\% MRSA colonization on a university campus, ${ }^{11}$ physicians who frequently treat students and student athletes (family practitioners, pediatricians, orthopedists, physical therapists, and sports medicine specialists) should be 
knowledgeable that "staph infections", including MRSA, is occurring in previously healthy youth. They must utilize a diagnosis based on culture and susceptibility testing rather than empirical treatment, especially in cases of extreme pain of routine-appearing sores and boils. In addition, physicians (and others involved in the healthcare of students) should be proactive in establishing and maintaining strong, regular communication with athletic facility directors, student health centers, coaches, intramural personnel, athletic trainers, staff, and faculty so that a synergistic atmosphere is created to foster awareness when a MRSA (or similar) infection has occurred in their population and can take effective surveillance and prevention measures for other affected individuals. ${ }^{16,30}$ For instance, we recommend that these individuals come together at the very point of likely retention - the social friendly environment in these recreational and athletic facilities - for focused education with visual stimuli (e.g. animations, video, or "stories" about what MRSA is and what it looks like) for today's modern student. Lastly, we urge all healthcare professionals in the university setting and the general public, to contact their local, regional, or state health department when they recognize an unusual number of students with Staphylococcus species infections, especially MRSA, that meets internal or external outbreak or severe criteria.

\section{ACKNOWLEDGEMENT}

The authors would like to thank the directors of the athletic \& recreational facility for their cooperation in allowing the study site and contributions of data on annual, monthly and daily facility numbers. We would also like to thank Texas State University, College of Health Professions, Clinical Laboratory Science (CLS) Program for financial and infrastructure support of this study. Additionally, we extend appreciation to CLS (Carmen Adams, PhD and Chair, Dave Falleur MEd MT) at Texas State University for their help and time with oversight of the purchasing and direction of the project. Additionally, a special thanks to Nathalie Austin MBA, MT (TX State CLS alumna), Chun Wang (DSHS) and Linda K. McDougal, M.S. (CDC) for providing confirmation of isolates. Finally, thanks to Susanna E. Schmink (CDC) for assisting in the delivery of data for PFGE confirmation.

\section{REFERENCES:}

1. Gorwitz RJ, et al. Changes in the prevalence of nasal colonization with Staphylococcus aureus in the United States, 2001-2004. J of Infect Dis 2008;197:1226-34.

2. Kallen AK, et al. Health care-associated invasive MRSA infections, 2005-2008. JAMA. 2010; 304(6);641-8.

3. Stanforth B, Krause A, Starkey C, Ryan T. Prevalence of community-associated methicillin-resistant Staphylococcus aureus in high school wrestling environments. J Env Health 2010;72(6).

4. CDC.gov [Internet]. Atlanta: Centers for Disease Control and Prevention. Available from http://www.cdc.gov/mrsa/. Accessed 2011 Feb 27.

5. Klevens M, et al. Invasive methicillin-resistant Staphylococcus aureus infections in the United States. JAMA 2007;298(15);1763-71.

6. Bartlett PC, Martin RJ, Cahill BR. Furunculosis in a high school football team. Am J Sports Med 1982;10:371-4.

7. Sosin DM, Gunn RA, Ford WL, Skaggs JW. An outbreak of furunculosis among high school athletes. Am J Sports Med 1989;17:828-32.

8. Stacey AR, Endersby KE, Chan PC, Marples RR. An outbreak of methicillin resistant Staphylococcus aureus infection in a rugby football team. Br J Sports Med 1998;32:153-4.

9. Lindenmayer JM, Schoenfeld S, O'Grady R, Carney JK. Methicillin resistant Staphylococcus aureus in a high school wrestling team and the surrounding community. Arch Intern Med 1998;158:895-9.

10. Felkner M, Rohde RE, Valle-Rivera AM, et al. Methicillinresistant Staphylococcus aureus nasal carriage rate in Texas county jail inmates. J Correctional Health Care 2007;13(4):289-95.

11. Rohde RE, Denham R, \& Brannon A. Methicillin resistant Staphylococcus aureus: Carriage rates and characterization of students in a Texas university. Clin Lab Sci 2009;22(3);17684.

12. Weiner, R. Methicillin-resistant Staphylococcus aureus on campus: A new challenge to college health. J American College Health 2008;56(4):347-50.

13. Bischoff WE, Wallis ML, Tuckert KB, et al. Staphylococcus aureus nasal carriage in a student community: Prevalence, clonal relationships, and risk factors. Infect Cont \& Hosp Epidemiology 2004;25:485-91.

14. Huang $\mathrm{H}$, Cohen $\mathrm{SH}$, King JH, et al. Injecting drug use and community-associated methicillin-resistant Staphylococcus aureus infection. Diagn Microbiol Infect Dis 2008;60(4):34750.

15. Nguyen DM, Mascola L, Bancroft E. Recurring methicillinresistant Staphylococcus aureus infections in a football team. Emerg Infect Dis 2005;11:526-32.

16. Barr B, Diamond PM, Felkner M. High school athletic departments as sentinel surveillance sites for community associated methicillin resistant staphylococcal infections. Texas Medicine 2006;56-61.

17. Centers for Disease Control and Prevention (CDC). Methicillin resistant Staphylococcus aureus infections among competitive sports participants - Colorado, Indiana, Pennsylvania, and Los Angeles County, 2000-2003. MMWR 2003;52:793-5.

18. Begier EM, Frenette K, Barrett NL, et al. Connecticut Bioterrorism Field Epidemiology Response Team. A highmorbidity outbreak of methicillin resistant Staphylococcus 
aureus among players on a college football team, facilitated by cosmetic body shaving and turf burns. Clin Infect Dis 2004;39:1446-53.

19. Borchardt SM, Yoder JS, Dworkin MS. Is the recent emergence of community-associated methicillin resistant Staphylococcus aureus participants in competitive sports limited to participants? Clin Infect Dis 2005;40:906-7.

20. Kazakova SV, Hageman JC, Matava M, et al. A clone of methicillin resistant Staphylococcus aureus among professional football players. N Engl J Med 2005;352:468-75.

21. Boyce J. Environmental contamination makes an important contribution to hospital infection. J Hosp Infect 2007;65:50-4.

22. Duckworth GJ, Jordens JZ. Adherence and survival properties of an epidemic methicillin-resistant strain Staphylococcus aureus compared with those of methicillin-sensitive strains. J Med Microbiol 1990;32:195-200.

23. Beard-Pegler MA, Stubbs, E, Vickery AM. Observations on the resistance to drying of staphylococcal strains. J Med Microbiol 1988;26:251-5.

24. Farrington M, Brenwald N, Haines D, Walpole E. Resistance to dessication and skin fatty acids in outbreak strains of methicillin-resistant Staphylococcus aureus. J Med Microbiol 1992;36:56-60.
25. Colbeck JC. Environmental aspects of Staphylococcal infections acquired in hospitals. AJPH. 1960;50:468-73.

26. Rihn J, et al. Community-acquired methicillin-resistant Staphylococcus aureus: An emerging problem in the athletic population. Am J Sports Med 2006;33(12);1924-9.

27. Bogar, CT. Trends in collegiate recreational sports facilities. The Sport Journal 2008;11(4). Available from http://www.thesportjournal.org/article/trends-collegiaterecreational-sports-facilities. Accessed 2011 Oct 3.

28. Bureau of Labor and Statistics. Economic news release: College enrollment and work activity of 2010 high school graduates. Available from http://www.bls.gov/news.release/hsgec.nr0.htm. Accessed 2011 Oct 10.

29. Carling PC, Barley JM. Evaluating hygienic cleaning in healthcare settings: What you do not know can harm your patients. Am J Infect Cont 2010 Jun;38(5 Suppl 1):41-50.

30. Rohde, RE. Methicillin Resistant Staphylococcus aureus (MRSA) Knowledge, Learning, and Adaptation: I Guess Everything Changes When it Happens to You - Their Stories. Saarbrücken, Germany: LAP Lambert Academic Publishing $\mathrm{GmbH} \&$ Co. 2011. [ISBN 978-3-8433-8225-0].

\section{Annual Meeting Abstract Deadline}

The deadline for abstracts for oral or poster presentations of research or case studies at the 2013 ASCLS Annual Meeting is April 1, 2013. Submission instructions and the proposal form may be found at www.ascls.org/?Educational_Events. The completed proposal form and abstract must be submitted electronically by the deadline. The 2013 Annual Meeting will be held July 30-August 3 in Houston, TX. Additional meeting information will be available at www.ascls.org/?Annual_Meeting. 